\title{
INTEGER INVARIANTS OF CERTAIN EVEN-DIMENSIONAL KNOTS
}

\author{
C. KEARTON
}

\begin{abstract}
Integer invariants of certain simple $\mathbf{Z}$-torsion-free $2 q$-knots, $q \geqslant 4$, are defined. It is shown that for $q \geqslant 5$, certain of these invariants must vanish, mod 2, if the knot is doubly-null-concordant.
\end{abstract}

Introduction. An $n$-knot is a locally flat piecewise-linear pair $\left(S^{n+2}, S^{n}\right)$, both spheres being oriented. The exterior $K$ of $k$ is the closure of the complement of a regular neighbourhood of $S^{n}$. A $2 q$-knot $k$ is simple if $K$ has the homotopy type of a circle below dimension $q$; or, equivalently, if $\pi_{i}(K) \cong \pi_{i}\left(S^{1}\right)$ for $1 \leqslant i<q$. Let $\tilde{K}$ be the universal cover of $K$; then duality theorems show that $H_{q}(\tilde{K})$ and $H_{q+1}(\tilde{K})$ are the only nontrivial homology groups of $\tilde{K}$ in such a case. If, in addition, $H_{a}(\tilde{K})$ has no $\mathbf{Z}$-torsion, then we refer to $k$ as being $\mathbf{Z}$-torsion-free. The orientations of $S^{2 q}$ and $S^{2 q+2}$ yield a canonical generator $t$ for $H_{1}(K) \cong H_{1}\left(S^{1}\right)$, via the Alexander and Poincaré duality isomorphisms, and the covering space action on $\tilde{K}$ makes $H_{q}(\tilde{K})$ and $H_{q+1}(\tilde{K})$ into $\Lambda$-modules, where $\Lambda=\mathbf{Z}\left[t, t^{-1}\right]$. Blanchfield duality yields a hermitian pairing into $\Lambda_{0} / \Lambda$, where $\Lambda_{0}$ is the field of fractions of $\Lambda$, which identifies $H_{q+1}(\tilde{K})$ as the conjugate dual of $H_{q}(\tilde{K})$. Conjugation in $\Lambda$ is the linear extension of $t \rightarrow t^{-1}$.

For $q \geqslant 4$, the $\mathbf{Z}$-torsion-free simple $2 q$-knots have been classified algebraically in [1] by their associated $F$-forms

$$
\left(\mathscr{E}(\tilde{K}), H_{q}(\tilde{K}), p_{q}(\tilde{K}),[,]_{\tilde{K}}, \tau\langle,\rangle_{\tilde{K}}\right)
$$

where $p_{q}(\tilde{K}): H_{q}(\tilde{K}) \rightarrow H_{q}(\tilde{K}) / 2 H_{q}(\tilde{K})=\mathscr{H}_{q}(\tilde{K})$ is the quotient map, $\mathscr{H}_{q+1}(\tilde{K})$ $=H_{q+1}(\tilde{K}) / 2 H_{q+1}(\tilde{K}), \Pi_{q+1}(\tilde{K})=\pi_{q+1}(\tilde{K}) / 2 \pi_{q+1}(\tilde{K})$, and

$$
\mathscr{E}(\tilde{K}): \mathscr{H}_{q}(\tilde{K}) \stackrel{\omega}{\mapsto} \Pi_{q+1}(\tilde{K}) \stackrel{h}{\mapsto} \mathscr{H}_{q+1}(\tilde{K})
$$

is a short exact sequence (s.e.s.) of $\Gamma$-modules $\left(\Gamma=\mathbf{Z}_{2}\left[t, t^{-1}\right]\right)$.

The Blanchfield pairing induces the nonsingular hermitian pairing

$$
{ }^{\theta}\langle,\rangle_{\tilde{K}}: \mathscr{H}_{q+1}(\tilde{K}) \times \mathscr{H}_{q}(\tilde{K}) \rightarrow \Gamma_{0} / \Gamma,
$$

Received by the editors March 20, 1984 and, in revised form, June 27, 1984

1980 Mathematics Subject Classification. Primary 57Q45.

Key words and phrases. High-dimensional knot, doubly-null concordant, hermitian pairing. 
which is related to the nonsingular hermitian pairing

$$
[,]_{\tilde{K}}: \Pi_{q+1}(\tilde{K}) \times \Pi_{q+1}(\tilde{K}) \rightarrow \Gamma_{0} / \Gamma
$$

by $[x, \omega(y)]_{\tilde{K}}={ }^{\theta}\langle h(x), y\rangle_{\tilde{K}}$ for all $x \in \Pi_{q+1}(\tilde{K}), y \in \mathscr{H}_{q}(\tilde{K})$.

To simplify notation, we shall identify $\mathscr{H}_{q+1}(\tilde{K})$ with the conjugate dual $\mathscr{H}_{q}(\tilde{K})^{*}$ and omit all mention of $\tilde{K}$ and $q$. Thus we have an s.e.s. of $\Gamma$-modules

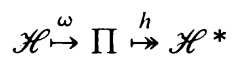

with pairings satisfying $[x, \omega(y)]=\langle h(x), y\rangle$.

1. The invariants. We shall be concerned with the special case of a $\Lambda$-module $H$ which is the direct sum of $\Lambda$-modules $H_{1}, \ldots, H_{n}$; each $H_{i}$ is annihilated by $p_{i}^{m}$, where $p_{1}, \ldots, p_{n}$ are distinct irreducible Laurent polynomials, with $p_{i}(t)=p_{i}\left(t^{-1}\right)$. Moreover, we assume that, $\bmod 2$, each $p_{i}$ is equal to the same polynomial $p$, also symmetric and irreducible. Then $\mathscr{H}=\mathscr{H}_{1} \oplus \cdots \oplus \mathscr{H}_{n}$ in the obvious notation. Since $\Gamma$ is a PID, $\mathscr{H}_{i}$ is a direct sum of $\Gamma$-modules $\mathscr{H}_{i, r}$ where $1 \leqslant r \leqslant m$ and each $\mathscr{H}_{i, r}$ is free over $\Gamma /\left(p^{r}\right)$. Similarly, $\mathscr{H}^{*}=\mathscr{H}_{1}^{*} \oplus \cdots \oplus \mathscr{H}_{n}^{*}$ and $\mathscr{H}_{i}{ }^{*}$ is the direct sum of modules $\mathscr{H}_{i, r}^{*}$.

In a $\Gamma$-module $M$ which is annihilated by $p^{m}$, let $\operatorname{ker} p^{r}=\left\{x \in M: p^{r} x=0\right\}$. Set

$$
M^{r}=\operatorname{ker} p^{r} /\left(\operatorname{ker} p^{r-1}+p \operatorname{ker} p^{r+1}\right) .
$$

Note that $\mathscr{H}^{* r}=\mathscr{H}_{1}{ }^{* r} \oplus \cdots \oplus \mathscr{H}_{n}{ }^{* r}$ and that $\mathscr{H}_{i}{ }^{* r}=\mathscr{H}_{i, r}^{*} / p \mathscr{H}_{i, r}^{*}$. For ker $p^{r} \subseteq \mathscr{H}^{*}$, let $\phi_{r}: \operatorname{ker} p^{r} \rightarrow \mathscr{H}^{* r}$ denote the quotient map. In $\Pi$, set $\Pi_{r}=h^{-1}\left(\operatorname{ker} p^{r}\right) \cap \operatorname{ker} p^{2 r}$. From now on we shall assume that the map $\left.\phi_{r} h\right|_{\mathrm{II}_{r}}: \Pi_{r} \rightarrow \mathscr{H}^{* r}$ is onto.

Let $r$ be fixed, $1 \leqslant r \leqslant m$. Choose a basis for $\mathscr{H}_{i}{ }^{* r}$, regarded as a vector space over the finite field $E=\Gamma /(p)$. Denote the union of these bases by $x_{1}, \ldots, x_{N}$, where it is understood that taking them in order gives first a basis for $\mathscr{H}_{1}^{* r}$, then a basis for $\mathscr{H}_{2}{ }^{* r}$, and so on. For each $j$, choose $z_{j} \in \Pi_{r}$ so that $\phi_{r} h\left(z_{j}\right)=x_{j}$. Define $a_{i j} \in E$ by $\left[p^{2 r-1} z_{i}, z_{j}\right]=b_{i j} / p, \psi\left(b_{i j}\right)=a_{i j}$, where $\psi: \Gamma \rightarrow E$ is the quotient map.

LEMMA. $a_{i j}$ is well defined.

Proof. The element $z_{j}$ may be replaced by $z_{j}^{\prime}=z_{j}+u_{j}+p v_{j}+\omega\left(w_{j}\right)$, where $h\left(u_{j}\right) \in \operatorname{ker} p^{r-1}, \quad h\left(v_{j}\right) \in \operatorname{ker} p^{r+1}$, and $u_{j}+p v_{j}+\omega\left(w_{j}\right) \in \operatorname{ker} p^{2 r}$. Note that $\left[p^{2 r-1} z_{i}^{\prime}, p v_{j}\right]=\left[p^{2 r} z_{i}^{\prime}, v_{j}\right]=0$, and so

$$
\begin{aligned}
{\left[p^{2 r-1} z_{i}^{\prime}, z_{j}^{\prime}\right] } & =\left[p^{2 r-1} z_{i}^{\prime}, z_{j}+u_{j}+\omega\left(w_{j}\right)\right] \\
& =\left[p^{2 r-1} z_{i}^{\prime}, z_{j}+u_{j}\right]+\left\langle p^{2 r-1} h\left(z_{i}^{\prime}\right), w_{j}\right\rangle \\
& =\left[p^{2 r-1} z_{i}^{\prime}, z_{j}+u_{j}\right] \text { since } p^{r} h\left(z_{i}^{\prime}\right)=0 \\
& =\left[p^{2 r-1}\left(z_{i}+u_{i}+p v_{i}\right), z_{j}+u_{j}\right]+\left\langle w_{i}, p^{2 r-1} h\left(z_{j}+u_{j}\right)\right\rangle \\
& =\left[p^{2 r-1}\left(z_{i}+u_{i}+p v_{i}\right), z_{j}+u_{j}\right] .
\end{aligned}
$$


Noting that $p^{r-1} u_{i}=\omega\left(\alpha_{i}\right)$, since $p^{r-1} h\left(u_{i}\right)=0$, and that $\left[p^{2 r} v_{i}, z_{j}\right]=\left[v_{i}, p^{2 r} z_{j}\right]$ $=\left[v_{i}, 0\right]=0$, we have

$$
\begin{aligned}
{\left[p^{2 r-1} z_{i}^{\prime}, z_{j}\right] } & =\left[p^{2 r-1}\left(z_{i}+u_{i}+p v_{i}\right), u_{j}\right]+\left[p^{2 r-1} z_{i}, z_{j}\right]+\left[p^{2 r-1} u_{i}, z_{i}\right] \\
& =\left\langle p^{r} h\left(z_{i}+u_{i}+p v_{i}\right), \alpha_{j}\right\rangle+\left[p^{2 r-1} z_{i}, z_{j}\right]+\left\langle p^{r} h\left(z_{j}\right), \alpha_{i}\right\rangle \\
& =\left[p^{2 r-1} z_{i}, z_{j}\right],
\end{aligned}
$$

since $h\left(z_{i}+u_{i}+p v_{i}\right), h\left(z_{i}\right) \in \operatorname{ker} p^{r}$.

Note that the matrix $A=\left(a_{i j}\right)$ is hermitian. If we make a different choice of basis for $\mathscr{H}_{i}{ }^{* r}$, then we obtain a transformation matrix $B$ which is a block diagonal matrix, and $A$ is replaced by $B A B^{*}$.

Write $A$ in block form as $\left(A_{i j}\right)$, where $i$ and $j$ run from 1 to $n$, corresponding to the choice of bases above. Then $B A B^{*}$ has the same form, $A_{i j}$ being replaced by $B_{i} A_{i j} B_{j}^{*}$, where $B=\operatorname{diag}\left(B_{1}, \ldots, B_{n}\right)$ and each $B_{i}$ is nonsingular. Set $\sigma_{i j}=\operatorname{rank}\left(A_{i j}\right)$; then these do not depend on the choice of bases, and so are invariants of the knot $k$. Note also that $\sigma_{i j}^{r}=\sigma_{j i}^{r}$, because $A$ is hermitian.

2. Doubly-null-concordant knots. An $n$-knot is doubly-knot-concordant if it is a cross section of the trivial $(n+1)$-knot. For a simple $\mathbf{Z}$-torsion-free $2 q$-knot $k$, $q \geqslant 5$, to be doubly-null-concordant, it is necessary and sufficient that its $F$-form have the following structure [2]:

(i) $H=H_{+} \oplus H_{-}$.

(ii)

$$
\begin{gathered}
\mathscr{E}^{=} \mathscr{E}_{+} \oplus \mathscr{E}_{-}, \text {where } \mathscr{E}_{-}=\mathscr{E}_{+}^{*}, \quad \text { and } \\
\mathscr{E}_{+}: \mathscr{H}_{+} \stackrel{\omega}{\mapsto} \Pi \stackrel{h}{\mapsto} \mathscr{H}_{-}^{*}, \quad \mathscr{E}_{-}: \mathscr{H}_{-} \stackrel{h^{*}}{\mapsto} \Pi^{*} \stackrel{\omega^{*}}{\mapsto} \mathscr{H}_{+}^{*} .
\end{gathered}
$$

(iii) $\Pi$ and $\Pi^{*}$ are self-annihilating under [ , ]; indeed, [, ] is given by the evaluation map $\Pi^{*} \times \Pi \rightarrow \Gamma_{0} / \Gamma$.

Note that, in such a case, $\Pi_{q+1}(\tilde{K})=\Pi \oplus \Pi^{*}$, and the $\omega, h$ of the Introduction are $\omega \oplus h^{*}, h \oplus \omega^{*}$, respectively.

THEOREM. Let $k$ be a doubly-null-concordant $2 q$-knot, $q \geqslant 5$, which satisfies the hypotheses of $\S 1$. Then $\sigma_{i i}^{r}$ is even, for all $i$ and $r$.

Proof. We know that $H$ splits as $H_{1} \oplus \cdots \oplus H_{n}$ and as $H_{+} \oplus H_{-}$, in both cases as a $\Lambda$-module. For each $i, H_{i}=\left\{x \in H: p_{i}^{m} x=0\right\}=\operatorname{ker} p_{i}^{m}$. If $x_{\varepsilon} \in H_{\varepsilon}$, then $p_{i}^{m}\left(x_{+}+x_{-}\right)=0$ if and only if $p_{i}^{m} x_{+}=0$ and $p_{i}^{m} x_{-}=0$. Thus $H_{i}=H_{i,+} \oplus H_{i,-}$, where $H_{i, \varepsilon}=H_{i} \cap H_{\varepsilon}, \varepsilon= \pm$, and so $\mathscr{H}_{\varepsilon}=\mathscr{H}_{1, \varepsilon} \oplus \cdots \oplus \mathscr{H}_{n, \varepsilon}$ in the obvious notation. Moreover, $\mathscr{H}_{\varepsilon}^{r}=\mathscr{H}_{1, \varepsilon}^{r} \oplus \cdots \oplus \mathscr{H}_{n, \varepsilon}^{r}$ for each $r$.

When choosing the basis $x_{1}, \ldots, x_{N}$ of $\mathscr{H}^{* r}$, we can therefore arrange for this to be the union of bases for $\mathscr{H}_{1,+}{ }^{* r}, \mathscr{H}_{1,-}{ }^{* r}, \mathscr{H}_{2,+}{ }^{* r}, \mathscr{H}_{2,-}{ }^{* r}, \ldots, \mathscr{H}_{n,+}{ }^{* r}, \mathscr{H}_{n,-}{ }^{* r}$ in that order. Since $\Pi$ and $\Pi^{*}$ are self-annihilating under the pairing, each diagonal block $A_{i i}$ of the 
hermitian matrix $A$ has the form

$$
\left(\begin{array}{cc}
0 & \beta_{i} \\
\beta_{i}^{*} & 0
\end{array}\right),
$$

and so $\sigma_{i i}^{r}=\operatorname{rank} \beta_{i}+\operatorname{rank} \beta_{i}^{*}=2 \operatorname{rank} \beta_{i}$ is even.

3. Examples. The first two examples are given in [1, p. 52]. Let $p_{1}(t)=t^{-1}-1+t$, $p_{2}(t)=t^{-1}-3+t$, and set $H=\Lambda /\left(p_{1}\right) \oplus \Lambda /\left(p_{2}\right)$ with generators $x_{1}, x_{2}$ for $\mathscr{H}^{*}$. Let $\Pi=\Gamma /\left(p^{2}\right) \oplus \Gamma /\left(p^{2}\right)$ with generators $z_{1}, z_{2}$ so that $h\left(z_{i}\right)=x_{i}$ for $i=1,2$. Define hermitian forms [ , ] and $\langle$,$\rangle on \Pi$ by

(i) $\left[z_{1}, z_{1}\right]=1 / p^{2}=\left[z_{2}, z_{2}\right],\left[z_{1}, z_{2}\right]=0$,

(ii) $\left\langle z_{1}, z_{1}\right\rangle=0=\left\langle z_{2}, z_{2}\right\rangle,\left\langle z_{1}, z_{2}\right\rangle=1 / p^{2}$.

In the first case, the matrix $A$ is $\left(\begin{array}{ll}1 & 0 \\ 0 & 1\end{array}\right)$, and in the second case it is $\left(\begin{array}{ll}0 & 1 \\ 1 & 0\end{array}\right)$. The invariants $\sigma_{i j}^{r}$ are

(i) $\sigma_{11}^{1}=\sigma_{22}^{1}=1, \sigma_{12}^{1}=0$;

(ii) $\sigma_{11}^{1}=\sigma_{22}^{1}=0, \sigma_{12}^{1}=1$.

Note that the first $F$-form belongs to a knot which is not doubly-null-concordant; taking $\mathscr{H}_{+}{ }^{*}=\left\langle x_{1}\right\rangle, \mathscr{H}_{-}^{*}=\left\langle x_{2}\right\rangle$, it is easy to see that the second $F$-form represents a doubly-null-concordant knot.

Defining the hermitian form in the obvious way, we can create $F$-forms giving rise to a matrix $A$ which is

(iii) $\left(\begin{array}{ll}1 & 1 \\ 1 & 0\end{array}\right)$, and

(iv) $\left(\begin{array}{ll}0 & 1 \\ 1 & 1\end{array}\right)$. Neither of the corresponding knots is doubly-null-concordant, by the Theorem.

\section{REFERENCES}

1. C. Kearton, An algebraic classification of certain simple even-dimensional knots, Trans. Amer. Math. Soc. 276 (1983), 1-53.

2. __ Doubly-null concordant simple even-dimensional knots, Proc. Roy. Soc. Edinburgh Sect. A 96 (1984), 163-174.

Department of Mathematics, University of Durham, Durham Dh1 3LE, England 